\title{
Characterization of cytoplasmic cyclin D1 as a marker of invasiveness in cancer
}

\author{
Noel P. Fusté ${ }^{1,4, *}$, Esmeralda Castelblanco $2,5,{ }^{*}$, Isidre Felip ${ }^{2,4, *}$, Maria Santacana 2,5 , \\ Rita Fernández-Hernández ${ }^{1,4}$, Sònia Gatius ${ }^{2,5}$, Neus Pedraza ${ }^{1,4}$, Judit Pallarés ${ }^{2,5}$, \\ Tània Cemeli ${ }^{1,4}$, Joan Valls ${ }^{3}$, Marc Tarres ${ }^{1,4}$, Francisco Ferrezuelo ${ }^{1,4}$, Xavier Dolcet ${ }^{2,4}$, \\ Xavier Matias-Guiu ${ }^{2,4,5}$ and Eloi Garí1,4 \\ 1 Department of Cell Cycle, Lleida, Catalonia, Spain \\ 2 Department of Oncological Pathology, Lleida, Catalonia, Spain \\ ${ }^{3}$ Department of Biostatistics and Epidemiology Unit of the Institut de Recerca Biomèdica de Lleida (IRBLLEIDA), Lleida, \\ Catalonia, Spain \\ ${ }^{4}$ Department of Basic Medical Sciences at University of Lleida, Lleida, Catalonia, Spain \\ ${ }^{5}$ Department of Pathology and Molecular Genetics at Hospital Universitari Arnau de Vilanova, Lleida, Catalonia, Spain \\ * These authors have contributed equally to this work \\ Correspondence to: Eloi Garí, email: eloi.gari@cmb.udl.cat
}

Xavier Matias-Guiu, email: fjmatiasguiu.lleida.ics@gencat.cat

Xavier Dolcet, email: dolcet@cmb.udl.cat

Keywords: cyclin D1, tissue array, cell invasion, metastasis, Pathology Section

Received: December 22, $2015 \quad$ Accepted: April 07, $2016 \quad$ Published: April 20, 2016

\section{ABSTRACT}

Cyclin D1 (Ccnd1) is a proto-oncogen amplified in many different cancers and nuclear accumulation of Cond1 is a characteristic of tumor cells. Cond 1 activates the transcription of a large set of genes involved in cell cycle progress and proliferation. However, Ccnd1 also targets cytoplasmic proteins involved in the regulation of cell migration and invasion. In this work, we have analyzed by immunohistochemistry the localization of Cond1 in endometrial, breast, prostate and colon carcinomas with different types of invasion. The number of cells displaying membranous or cytoplasmic Cond1 was significantly higher in peripheral cells than in inner cells in both collective and pushing invasion patterns of endometrial carcinoma, and in collective invasion pattern of colon carcinoma. Also, the cytoplasmic localization of Ccnd1 was higher when tumors infiltrated as single cells, budding or small clusters of cells. To evaluate cytoplasmic function of cyclin D1, we have built a variant (Ccnd1-CAAX) that remains attached to the cell membrane therefore sequestering this cyclin in the cytoplasm. Tumor cells harboring Cond1-CAAX showed high levels of invasiveness and metastatic potential compared to those containing the wild type allele of Cond1. However, Ccnd1CAAX expression did not alter proliferative rates of tumor cells. We hypothesize that the role of Cond1 in the cytoplasm is mainly associated with the invasive capability of tumor cells. Moreover, we propose that subcellular localization of Ccnd1 is an interesting guideline to measure cancer outcome.

\section{INTRODUCTION}

Cyclin D1 (Ccnd1) is among the 10 proto-oncogenes most frequently amplified in cancer [1]. Indeed, Ccnd1 was first characterized by studies of gene amplification as the gene affected by a chromosome inversion in parathyroid adenoma (PRAD1) and by a translocation $\mathrm{t}(11: 14)$ in B-Cell Lymphomas (BCL-1) [2]. Alternatively, Cend1 was also isolated from a human glioblastoma library in a yeast genetic selection using G1-cyclins depleted cells [3]. The importance of Ccnd1 as an oncogene has been extensively reported. For instance, overexpression of 
Cend 1 in the mammary gland is sufficient for the induction of mammary carcinoma [4]. This cyclin is a regulatory subunit of the cyclin-dependent kinases $\mathrm{Cdk} 4 / 6$ that are positive regulators of cell proliferation [5][6][7]. The Ccnd $1 \cdot \mathrm{Cdk} 4$ complex phosphorylates the transcriptional repressor $\mathrm{pRB}$ releasing the $\mathrm{E} 2 \mathrm{~F}$-dependent transcription required for $\mathrm{S}$ phase entry [8]. Alternatively, Ccnd1 regulates transcription of a different set of genes in a Cdkindependent manner [9]. In both cases, the transcriptional changes induced by Ccnd1 accumulation in the nucleus trigger cell transformation [10].

Ccnd1 has been also associated with tumor invasion and metastasis in clinical studies. For instance, overexpression of Ccnd1 is connected with metastatic prostate cancer to bone [11]. Moreover, knock down of Ccnd1 in xenografted lung adenocarcinoma cells abrogates its metastatic potential [12]. Consistently, it is also established that Ccnd1 enhances cell migration and invasion as $\mathrm{Ccnd}^{-/-}$cells show a reduction of their potential of invasiveness [13][14]. Interestingly, different works suggest that Ccnd 1 is not restricted to the nucleus but is also associated to the cytoplasmic membrane, where it can activate cytoplasmic targets involved in cell invasive potential [15][16][17][18][19]. Cytosolic localization of Cend1 has been described in different tumors. For instance, an elevated expression of cytoplasmic Ccnd1 has been detected by IHC in lymph node metastases originated from prostate cancer and is associated with poor survival [20]. However, the possible correlation between cytoplasmic Cend1 localization in neoplastic tissues and its ability to enhance invasion and metastasis has not been analyzed yet. In this work, we show that Cond1 displays an asymmetric pattern of localization in different cancers, being more cytoplasmic and membranous in the peripheral and invasive regions of the neoplastic tissues. We have mimicked this feature in the cells by using a membrane-attached variant of Ccnd1. We have observed that the sequestration of Ccnd1 in the membrane enhances cell invasion and metastasis without affecting cell proliferation. In the same way as the nuclear localization of Cend1 is a signal of proliferation in the IHC analyses, we hypothesize that cytoplasmic localization could be a marker of invasiveness.

\section{RESULTS}

\section{Cytoplasmic-membranous Cend1 expression in human tumor tissue sections}

To evaluate Ccnd1 localization, we performed IHC analysis of Cend 1 on different tumoral types. First, we analyzed 55 samples of endometrioid endometrial carcinoma displaying different types of invasion (collective, pushing, glandular, MELF, single cell/small cell clusters, and blood vessel invasion; see Table 1). As we show in Figure 1A, the different types of invasion showed differential patterns of Cend1 localization. Cytoplasmic staining seems reticular and not diffuse, and staining in the plasma membrane of certain cells is also observed. According with previous works showing that Ccnd1 is localized in cell membranes [16], we have referred the non-nuclear Ccnd1 as cytoplasmicmembranous localization. Ccnd1 cytoplasmic and membranous expression in collective and pushing patterns of endometrial samples was significantly higher in peripheral cells in comparison with the inner cells $(P=$ $<0.00001$ and $P=0.0004$ respectively; Figure 1B). Single cell/small cell cluster, MELF and glandular patterns had the highest Ccnd1 cytoplasmic-membranous expression of all invasion types.

In breast adenocarcinoma, cytoplasmic-membranous Ccnd1 protein expression was evaluated in 50 samples displaying different types of invasion (collective, glandular, indian-file). All invasion types showed high expression of cytoplasmic-membranous Cend1 but no differences between peripheral and inner cells in the collective invasion pattern $(P=0.18)$ (Figure $1 \mathrm{C}$; see also Supplementary Figure 1A).

In prostatic adenocarcinoma, cytoplasmicmembranous Ccnd1 protein expression was evaluated in 50 samples, with different types of Gleason grade $(3,4,5)$. Cytoplasmic-membranous Ccnd 1 expression increased in parallel with the Gleason grade and, the higher expression occurred in pT3, that is, when tumor extends beyond the prostate (Figures $1 \mathrm{D}$, trend test $P=0.003$; see also Supplementary Figure 1C).

In colon adenocarcinoma, cytoplasmic-membranous Ccnd1 protein expression was evaluated in 50 samples, with different types of invasion (collective, pushing, budding, glandular). In the collective pattern, cytoplasmicmembranous Ccnd1 expression was significantly higher in peripheral cells in comparison with inner cells $(P=0.01)$. In the pushing pattern, the difference between peripheral and inner cells was not statistically significant $(P=$ 0.15 ). The budding pattern had the highest cytoplasmicmembranous Ccnd1 expression of all invasion types. Interestingly, the expression of Ccnd1 in the cytoplasm and membrane of glandular cells was very low (Figure 1E; see also Supplementary Figure 1B).

Our results show that cytoplasmic-membranous staining for CcndD1 is weaker than nuclear, and a clear membrane signal is only observed in a small fraction of tissue cells. Probably, this result is not uncommon considering that the localization of Ccnd1 in the membrane of cultured cells was also detected only in a fraction of cells [16]. Three hours after seeding on fibronectin, mouseembryonic fibroblasts and tumor-endometrial cells showed Ccnd1 in the membrane of spreading cells (Supplementary Figure 2A). MFE cells reveal slightly membrane colocalization of Ccnd1 with RalA (Supplementary Figure 
Table 1: Definition of the different types of invasion evaluated in the tumours

\begin{tabular}{|c|c|c|c|c|}
\hline Invasive Type & ECET $^{1}$ & BAC $^{2}$ & PAC $^{3}$ & $\mathrm{CAC}^{4}$ \\
\hline $\begin{array}{l}\text { Collective invasion } \\
\text { Groups of cells invade the peritumoral stroma while maintaining cell-cell } \\
\text { contacts. }\end{array}$ & $x$ & $\mathrm{x}$ & & $\mathrm{x}$ \\
\hline $\begin{array}{l}\text { Single-cells/single cluster of cells } \\
\text { In the absence of cell-cell adhesion, tumour cells invade as single cells or small } \\
\text { cell clusters. }\end{array}$ & $\mathrm{x}$ & & & \\
\hline $\begin{array}{l}\text { Budding } \\
\text { Presence of individual cells and small clusters of tumour cells at the invasive } \\
\text { front of carcinomas. }\end{array}$ & & & & $\mathrm{x}$ \\
\hline $\begin{array}{l}\text { MELF } \\
\text { The microcystic, elongated and fragmented (MELF) pattern include the presence } \\
\text { of small dilated glands lined by cuboidal or flattened cells with eosinophylic } \\
\text { cytoplasm. }\end{array}$ & $x$ & & & \\
\hline $\begin{array}{l}\text { Invasive glands } \\
\text { Invasive glands often have a slit-like appearance. }\end{array}$ & $x$ & $x$ & & $\mathrm{x}$ \\
\hline $\begin{array}{l}\text { Vascular invasion } \\
\text { When tumour cells are noted within or attached to the wall of the vascular space } \\
\text { lined by flattened endothelial cells. }\end{array}$ & $x$ & & & \\
\hline $\begin{array}{l}\text { Pushing border invasion } \\
\text { Characterized by a cohesive tumour growth with well-delineated but infiltrating } \\
\text { borders and "pushing" margins. }\end{array}$ & $x$ & & & $\mathrm{x}$ \\
\hline $\begin{array}{l}\text { Indian-file } \\
\text { The tumour cells spread between the collagen bundles in a single line. }\end{array}$ & & $x$ & & \\
\hline Gleason 3, 4, 5 & & & $x$ & \\
\hline $\begin{array}{l}\text { pT3 } \\
\text { Tumour extends beyond the prostate }\end{array}$ & & & $\mathrm{x}$ & \\
\hline
\end{tabular}

$\mathrm{ECET}^{1}$, Endometrial carcinoma of endometrioid type; $\mathrm{BAC}^{2}$, Breast adenocarcinoma; PAC ${ }^{3}$, Prostate adenocarcinoma, $\mathrm{CAC}^{4}$, Colorectal adenocarcinoma.

2B). The presence of Ccnd1 only in the membrane of spreading cells agrees with the role of Ccnd1. Cdk 4 in the regulation of Rho and Ral GTPases activity during adhesion and migration processes [14].

Since membranous-cytoplasmic accumulation of Ccnd1 was seen at the periphery of nests in collective and pushing invasion patterns of endometrial carcinoma samples, but also in correlation with Gleason grade, and pT3 in prostatic cancer, we selected endometrial and prostatic cancer as models to further validate the role of Cendl in invasion.

\section{The addition of a farnesylation motif to Cend1 enhances its localization to the membranes}

We have previously described that $\mathrm{Ccnd} 1 \cdot \mathrm{Cdk} 4$ binds to Rgl2 that is a GEF of the Ral GTPases [18]. We hypothesized that $\mathrm{Ccnd} 1 \cdot \mathrm{Cdk} 4$ promotes Ral activation, and consequently cell invasion, through the regulation of $\mathrm{Rg} 12$. For Ral activation, $\mathrm{Rgl} 2$ has to be recruited to the membrane by the GTPase Ras. An Rgl2 variant containing the membrane-anchor motif of K-Ras promotes constitutive activation of Ral GTPase [21]. Then, to test whether the forced association of Ccnd1 in the membrane could induce invasion of tumor cells, we have built a Ccnd1 with the same membrane-anchor motif of K-Ras fused at the C-terminus. This anchor motif contains a polybasic domain that drives the protein to the negatively charged membrane and a CAAX motif with a cysteine residue to attach a farnesyl tail [22] (Figure 2A). The endometrial cells were infected with lentiviruses containing either HA-Ccnd1 or HA-Ccnd1-CAAX alleles and were grown in complete medium. After five days, cells were fixed and processed for immunofluorescence using the rat 3F10 anti-HA antibody to visualize the localization of the exogenous Ccnd1. We observed that the wild type allele was localized in both the nucleus and the cytoplasm whereas the Ccnd1-CAAX allele was mostly localized at the membrane and cytoplasm (Figure 2B). Moreover, we determined that only Ccnd1-CAAX widely co-localizes in the membrane with the GTPase RalA that naturally contains a farnesylation motif and is localized in the membrane. These results indicate that the addition of K-Ras farnesylation motif to Cend 1 can efficiently target it to the membrane. 

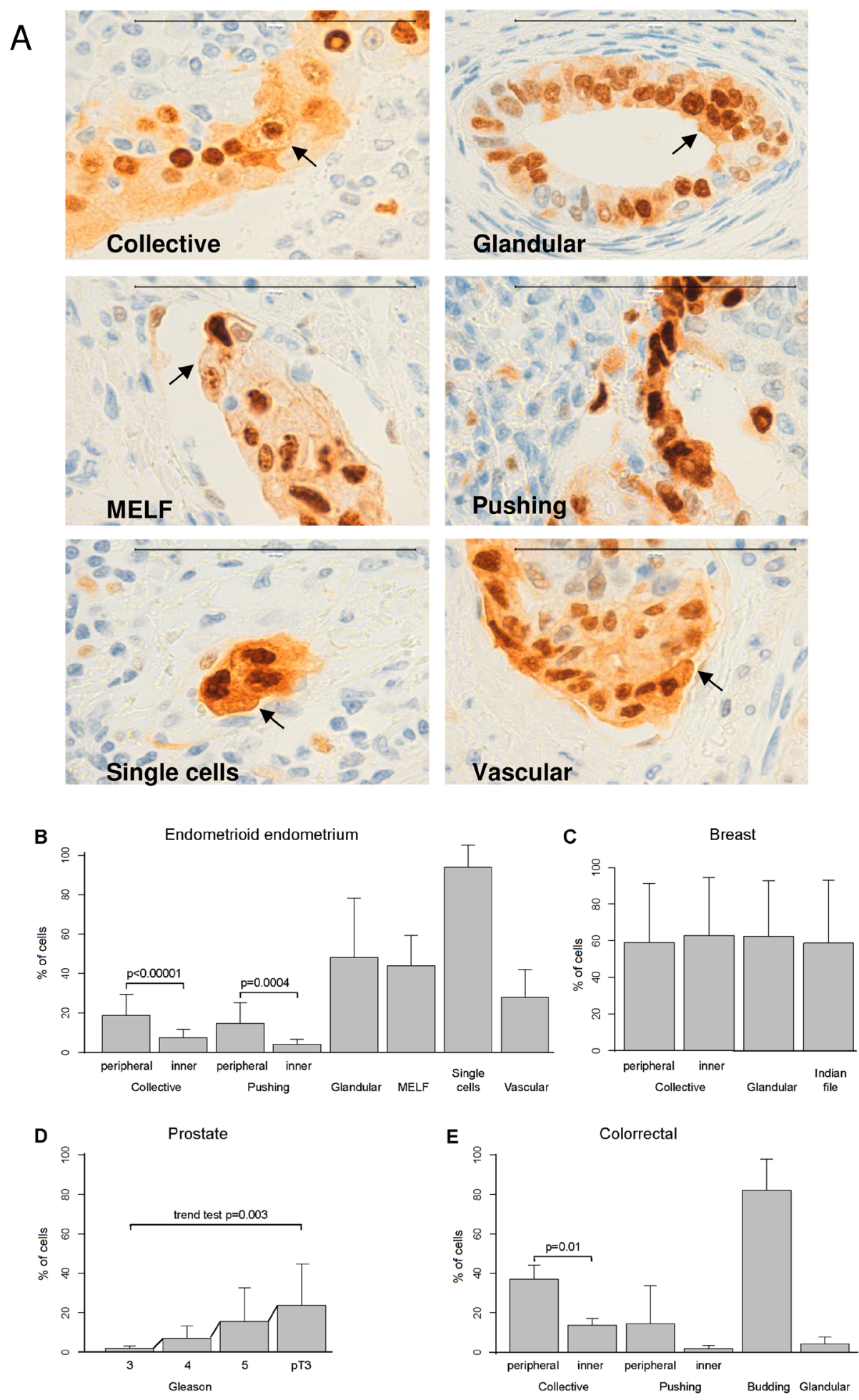

Figure 1: Membranous-cytoplasmic Cend1 expression at the invasive front is higher in peripheral cells, in large invasive cell clusters or in specific types of invasion. A. Representative images showing Ccnd1 expression in endometrioid

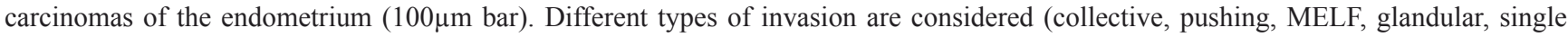
cells/small cluster of cells, and vascular). Arrows indicate Ccnd1 stain in the membrane. Evaluation of the differences in membranouscytoplasmic Ccnd1 expression among the different types of invasion in endometrioid endometrial carcinomas B., ductal breast carcinoma C., prostatic carcinoma according to Gleason grade or invasion beyond the prostate (pT3) D. and colonic carcinoma E. Bars represent mean percentages of positivity and segments one standard deviation. Significant differences between selected pairs are shown with their corresponding $\mathrm{p}$-value, as computed with the linear mixed models. For prostate, $p$-value to evaluate the increasing trend is shown. 
To confirm this result, we analyzed subcellular localization of Cend1 and Ccnd1-CAAX alleles by cell fractionation. Endometrial cells (Ishikawa) were infected with virus containing Ccnd1 or Ccnd1-CAAX and processed for cell fractionation using a Subcellular Protein Fractionation kit (Thermo Scientific-Pierce; 78840). We obtained a soluble fraction containing cytosolic and nuclear soluble proteins and a membrane fraction as indicated by the reference proteins (Figure 2C). We observed that the presence of Ccnd1-CAAX was extensively enriched in the membrane fraction (Figure 2C). Interestingly, the endogenous and exogenous wild type Ccnd 1 exhibited also a reduced portion of the protein in the membrane fraction in accordance with previous
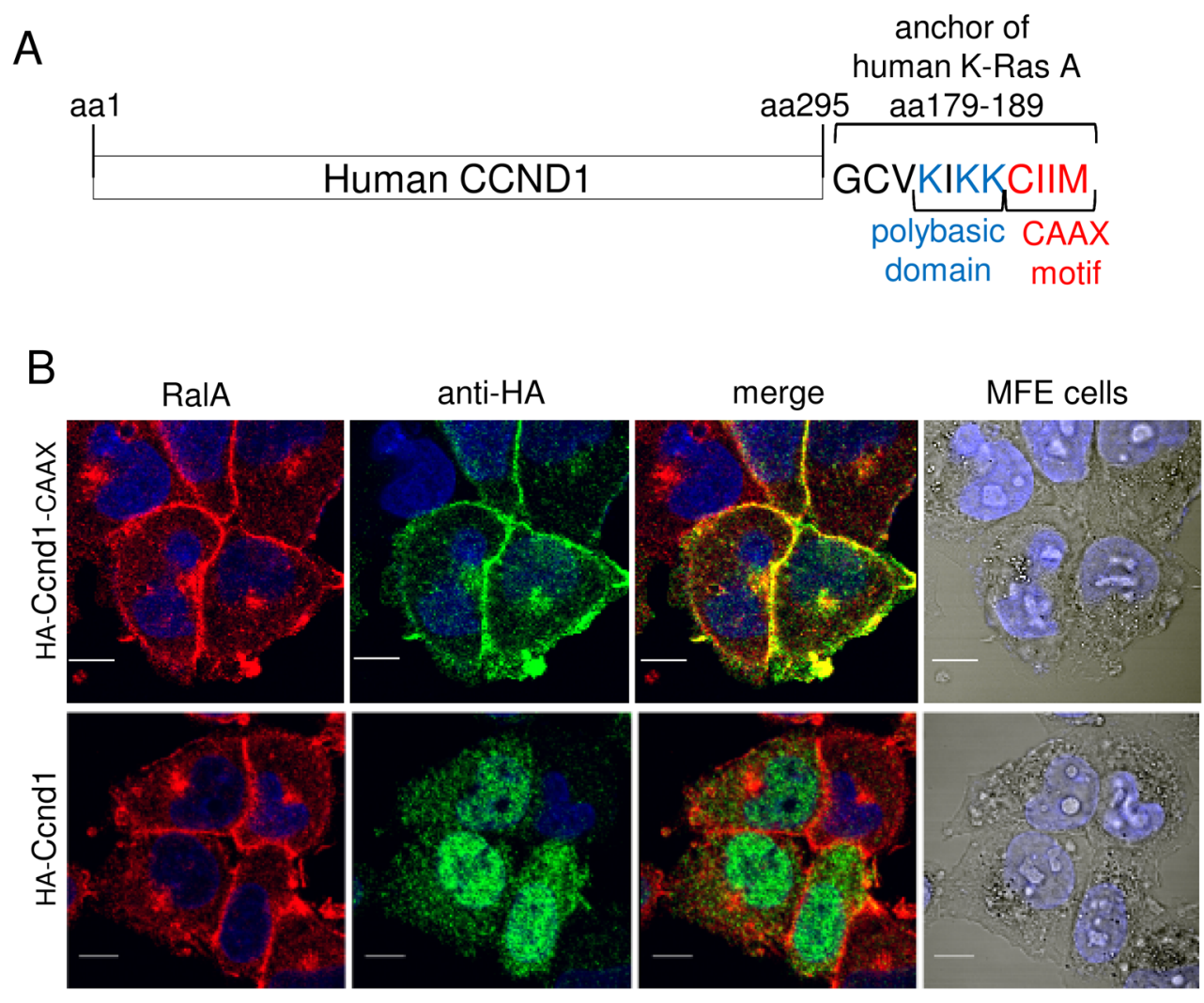

C

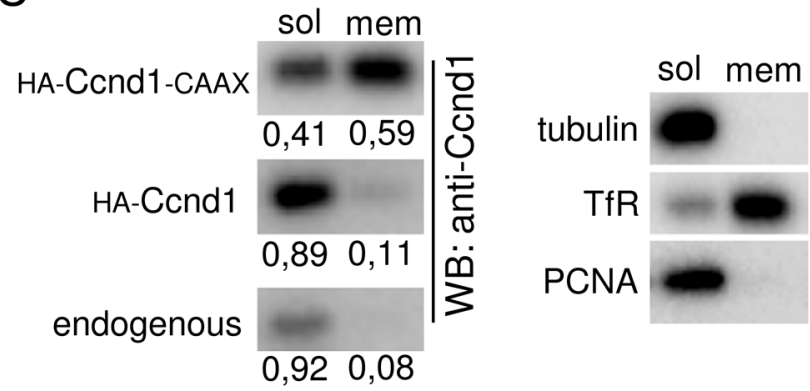

$\mathrm{D}$

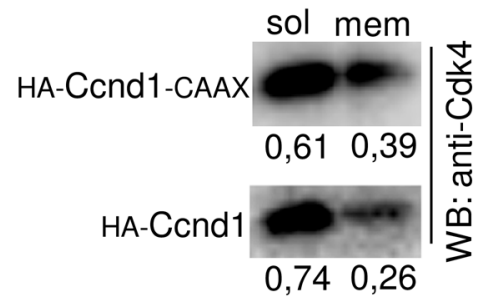

Figure 2: Ccnd1-CAAX is localized in cell membranes of tumor cells. A. Schematic representation of C-terminal fusion of the anchor domain of human K-Ras with Ccnd1 protein. B. MFE cells infected with HA-Ccnd1 or HA-Ccnd1-CAAX or an empty vector were fixed in $4 \%$ paraformaldehyde and permeabilized with $0.2 \%$ triton-100X. Images were acquired by confocal microscopy (10 $\mu \mathrm{m}$ bar). Nuclei were stained with Hoescht (blue). The antibodies used were anti-HA (rat monoclonal 3F10, green) and anti-RalA (mouse monoclonal, red). C. Ishikawa cells infected with HA-Ccnd1 or HA-Ccnd1-CAAX were submitted to subcellular fractionation (see "Materials and Methods"). Fractions were analyzed by immunoblotting to detect Cend1 in soluble and membrane fractions. Quantification of Ccnd1 levels are shown at the bottom of the panel. Tubulin as a cytosol marker, Transferrin Receptor as a membrane marker and PCNA as a nucleoplasm marker were used to control fractionation. D. Cdk4 distribution in soluble and membrane fractions from the experiment in C. Quantification of Cdk4 levels are shown at the bottom of the panel. 
works suggesting that Ccnd1 interacts with membraneassociated proteins as filaminA or RalA [16][18]. In these works, it has been suggested that cytoplasmic functions of Ccnd1 are Cdk4-dependent. For that reason, we have tested whether Cdk4 was also present in the membrane fraction (Figure 2D). As expected, a portion of Cdk4 was in the membrane and it was also enriched after Ccnd1CAAX expression. All these results indicate that the expression of Cend1-CAAX promotes the accumulation of Ccnd1-Cdk4 complexes in the membrane.
The sequestration of Cend1 in the membrane increases the invasiveness of tumor cells but does not change proliferation in those cells

Our study of Cend1 localization in cancer tissues suggested that the cytoplasmic localization of Ccnd1 may be related to the invasiveness of tumor cells (see above). It has also been reported that Ccnd 1 could have cytoplasmic substrates involved in the regulation of cell migration and invasion [16][18]. For these reasons, we sought to

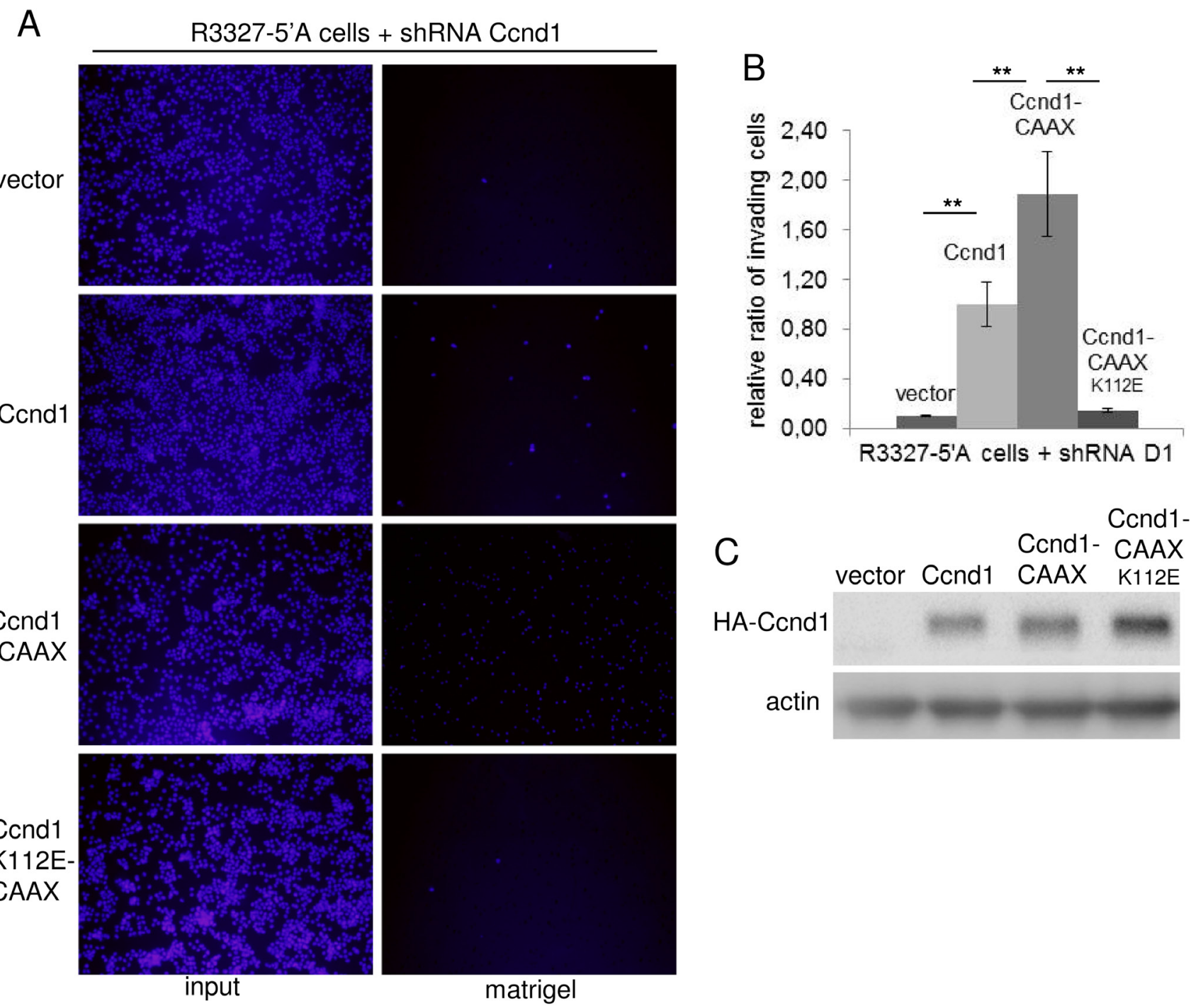

Figure 3: The membrane-associated Cend1 enhances invasiveness of tumor cells. A. R3327-5'A rat cells were infected with interference shRNA against rat Ccnd1 (shD1, Sigma). Cells expressing the shRNA were further infected with human Ccnd1, Ccnd1$\mathrm{CAAX}, \mathrm{Ccnd} 1_{\mathrm{K} 112 \mathrm{E}}-\mathrm{CAAX}$ or with an empty lentiviral vector. For invasion assays, $5 \times 10^{4}$ co-infected cells were seeded in 24 -well transwell filters previously coated with matrigel, and allowed to invade for 24 hours. After this time all cells were fixed and stained with Hoescht -input-. Then, the remaining cells at the bottom of the filter were washed and Matrigel-embedded cells were counted -matrigel- . B. Relative values from the experiment in A are expressed in mean \pm sem. Data are from at least three independent experiments. Significance values were determined by one way ANOVA and Holm T-statistic post-test $(* * p<0.01)$. C. Immunoblot showing the expression of HA-Ccnd1 in the co-infected cells. Actin was used as a loading control. 
analyze whether the membrane-associated allele of Ccnd1 could significantly increase the invasiveness. We infected R3327-5'A rat tumor cells that had endogenous Cend1 downregulated by RNA interference with lentiviruses containing human HA-Ccnd1, HA-Ccnd1-CAAX or HA-Ccnd $1_{\mathrm{K} 112 \mathrm{E}}-\mathrm{CAAX}$ or empty vector. After five days growing in complete medium, those cells were processed to reveal "in vitro" their invasive potential. We observed that the expression of Ccnd1-CAAX significantly induced the invasiveness of the R3327-5' A cells in comparison with the expression of the wild type allele or the empty vector (Figure 3A and 3B). It has been previously reported that Ccnd1 enhanced cell invasiveness by a Cdk4dependent mechanism [14]. In accordance with that result, we also remarked that the expression of the inactive allele Cend $1_{\mathrm{K} 112 \mathrm{E}}-\mathrm{CAAX}$ was unable to promote cell invasion. All these results suggest that the sequestration of an active complex Ccnd1-Cdk4 in the membrane increases the invasiveness of tumor cells.

It has been reported that overexpression of Ccnd1 reduces doubling-cell time and increases clonogenic and anchorage-independent growth in transformed cells [23][24]. Moreover, Ccnd1 alleles or mutants that enhance nuclear accumulation increase clonogenic growth capability over the wild type allele, even in nontransformed cells [25][26]. Then, we questioned whether the membrane-associated allele of Ccnd1 could estimulate cell proliferation. To test this hypothesis, MFE cells were infected with lentiviruses containing HA-Ccnd1 or HACcnd1-CAAX or empty vector. After growing, those cells were handled to determine the proliferation rate and clonogenic and anchorage-independent growth. The expression of Cend1-CAAX did not significantly change the proliferation rate of MFE cells compared to the wild type Ccnd1 (Supplementary Figure 3A). Also we noted that neither the clonogenic nor non-adherent growth was

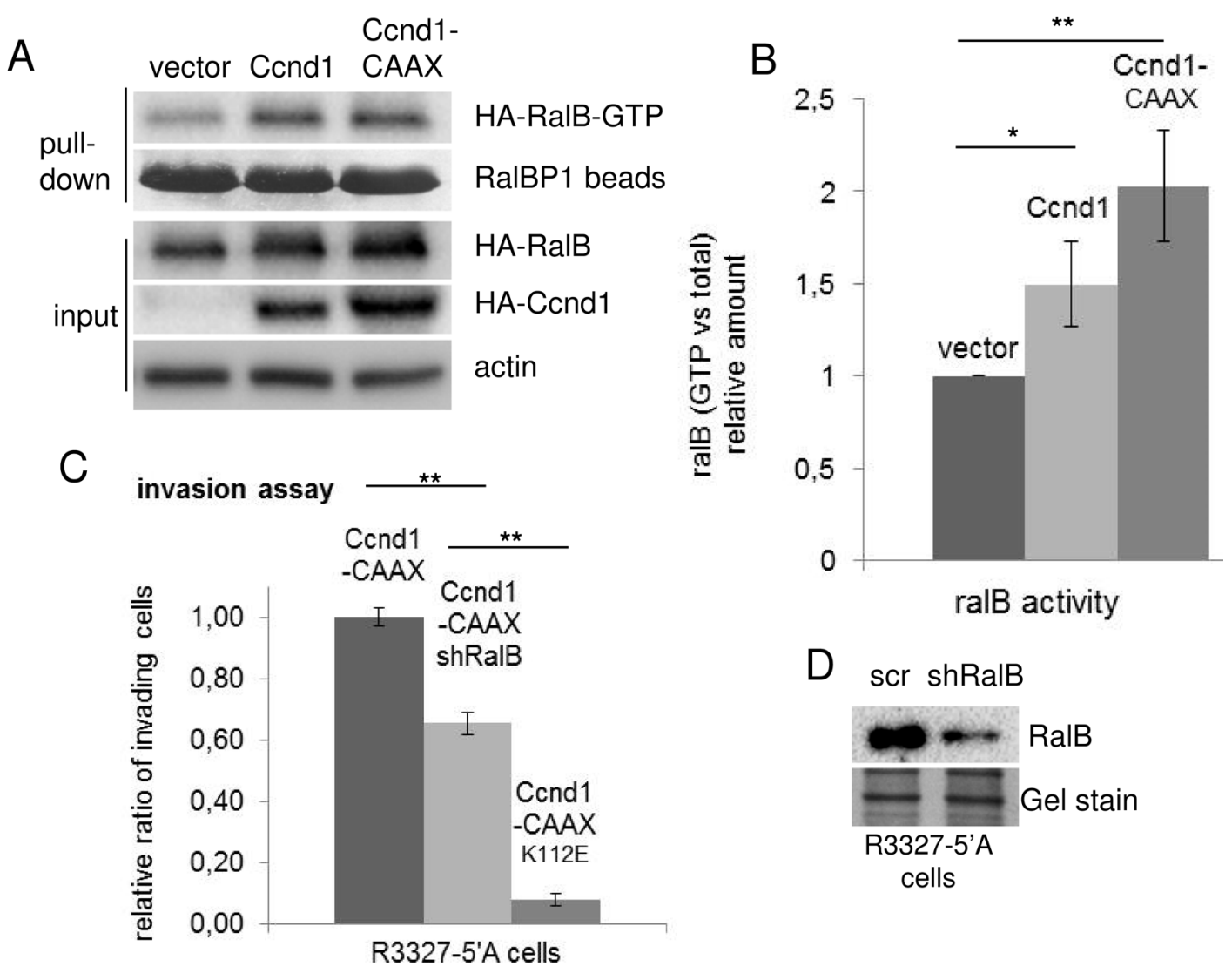

Figure 4: The membrane-associated Ccnd1 promotes Ral-GTPase activation. A. HEK293T cells were co-transfected with HA-RalB and either HA-Ccnd1, or HA-Ccnd1-CAAX or an empty vector as a negative control. Twenty-four hours after transfection, active RalB-GTP was affinity purified with RalBP-beads from cell lysates and detected by immunoblotting with anti-HA antibody. HA-D1, HARalB and HA-RalB-GTP amount from a representative experiment is shown. RalBP-beads and actin were used as loading controls. B. The experiment was independently repeated four times. Relative mean values \pm sem for the HA-RalB-GTP/ total HA-RalB ratio are plotted. Significance values were determined by one way ANOVA and Holm T-statistic post-test $\left({ }^{*} p<0.05 ; * * p<0.01\right)$. C. R3327-5'A rat cells were infected with interference shRNA against RalB (shRalB) or with scramble (scr) as a control. Relative invasion values are expressed in mean \pm sem. Data are from three independent experiments. Significance values were determined by one way ANOVA and Holm T-statistic post-test $(* * p<0.01)$. D. Immunoblot showing the levels of expression of RalB in (C). Gel stain was used as a loading control. 
modfified by the expression of Ccnd1-CAAX in MFE cells (Supplementary Figure 3B and 3C). These results suggest that sequestration of Ccnd1 in the membrane does not modify any form of growth in tumor cells.

\section{The attachment of Cond1 in the membrane promotes RalGTPase activation}

In a previous report we described that cyclin D1 enhanced cell motility through the activation of RalGTPases [18]. Then, we expected that the attachment of Ccnd1 to the membranes would ameliorate the activation of RalGTPases and consequently the efficiency of cell invasion. To analyze this possibility, we have coexpressed HA-Ccnd1 or HA-Ccnd1-CAAX with HA-RalB in HEK293T cells and then, we determined the levels of the HA-RalB loaded with GTP in those cells. The expression of Ccnd1-CAAX enhanced RalB activity better than the expression of Ccnd1, even though the increment

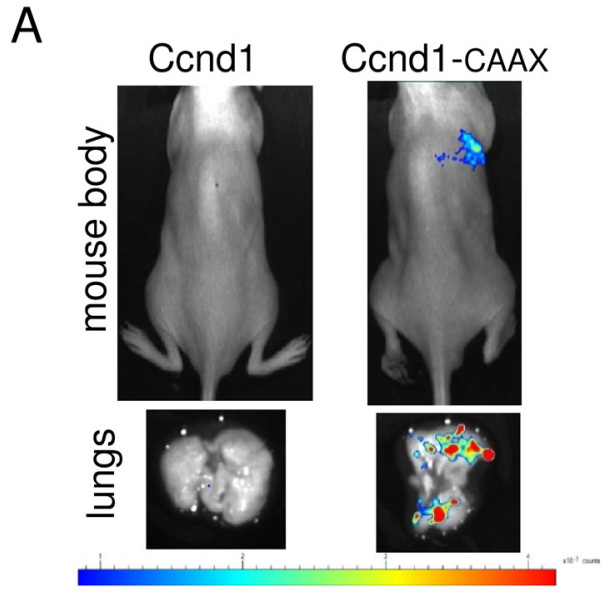

\section{C}

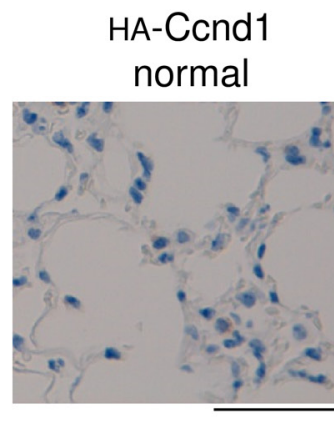

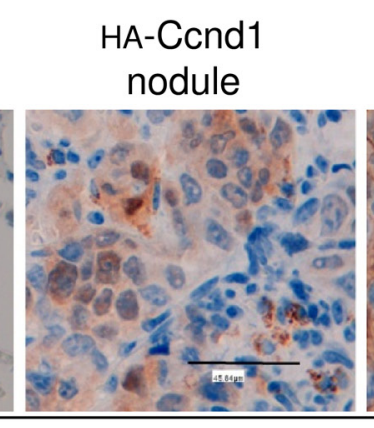

anti-HA in RalB activity was not statistically significant (Figure $4 \mathrm{~A}$ and $4 \mathrm{~B}$ ). Since the membrane attachment of Ccnd1 was able to appreciably improve the invasiveness of tumor cells, we tested whether the improvement in invasion was RalB-dependent. The down-regulation of RalB by shRNA in R3327-5'A cells expressing Ccnd1-CAAX partially reduced the invasion capacity of these cells (Figure 4C and 4D). Our data suggest that membrane-associated Ccnd1 promotes invasion in some extent through Ral activation, but it may have other interactors or substrates than Ral GTPases to completely explain the role of Ccnd1 in the membrane.

\section{The presence of Cend 1 attached to the membrane of tumor cells stimulates the tumor invasion and metastasis}

Ccnd1 has been described as an inductor of metastasis in mice and in clinical studies [27][12][11].

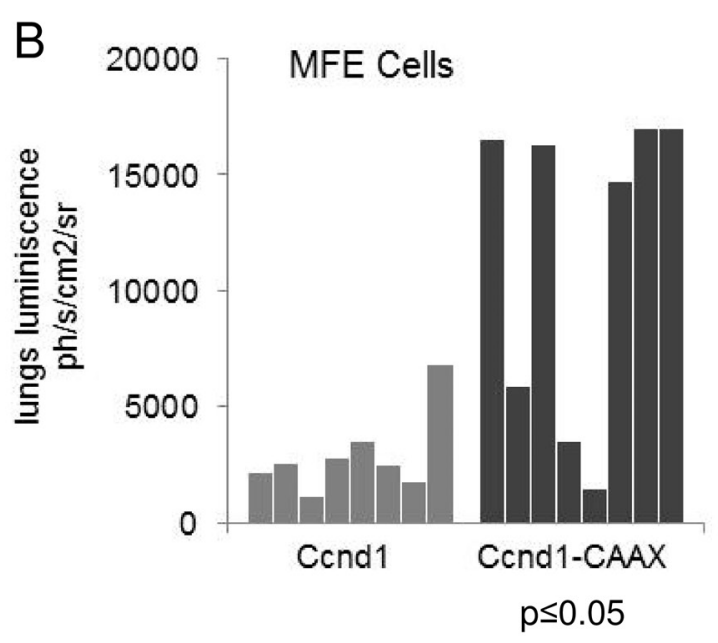

HA-Ccnd1-CAAX

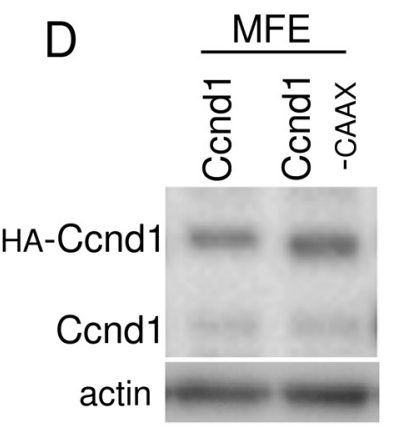

Figure 5: The membrane-associated Ccnd1 enhances lung metastatic activity of endometrial tumor cells. A. MFE cells stably expressing luciferase were used for metastasis assay. Cells harboring Cend1 or Ccnd1-CAAX were injected in SCID-hairless mice. Four weeks after injection, animals expressing Ccnd1-CAAX showed lung metastasis. Representative images of the body luminescence (top) and lungs luminiscence (bottom) are shown. B. The number of lung nodules from eight animals in A was inferred from luminescence and represented. Significance values were determined by a Mann-Whitney test. C. Representative HA-immunohistochemistry images of lung nodules are shown. D. MFE cells expressed similar amounts of Ccnd1 or Ccnd1-CAAX. A protein extract was obtained from the cells before injection to the mice, and the amount of Ccnd1 was analyzed by immunoblot. Actin was used as a loading control. 
To measure the relevance of the membrane-attachment of Ccnd1 in the induction of metastasis "in vivo", we injected MFE cells expressing Ccnd1-CAAX or Cend1 directly to the bloodstream of 8-weeks-old nude mice. Animals were sacrificed four weeks after injection, their lungs examined for metastatic growth and the presence of nodules in the lungs was calculated (Figure 5A and 5B). We observed that the expression of Ccnd1-CAAX significantly stimulated MFE-dependent metastasis (six out of the eight animals exhibited apparent nodules). After four weeks, only one animal manifested metastasis when the wild type allele of Ccnd1 was expressed in MFE cells. We confirmed the presence of the MFE cells in the nodules by revealing HA signal in the tissues, HA-Ccnd1-CAAX was detected in the membrane of cells (Figure 5C). Note that the abundance of metastases in the samples expressing Ccnd1-CAAX versus Ccnd1 was not due to differences in the expression levels (Figure 5D). Similar results were obtained using prostatic cancer cells (Supplementary Figure 4). Overall, this result suggests that the presence of Ccnd 1 in the cell membrane of tumor cells may be a marker of poor prognosis. This hypothesis is consistent with the data about cancer tissues analyzed in the first part of this paper.

\section{DISCUSSION}

The molecular mechanisms involved in both individual and collective tumor cell invasion are not fully understood. From the histopathological viewpoint, there are many different patterns of invasion, which frequently coexist in different areas of the same tumor. Some tumor types show specific patterns of invasion, which are not seen in other types of cancer. This phenomenon is clearly represented by endometrial cancer, which has a peculiar type of invasion (MELF, characterized by microcystic, elongated, and fragmented tumor cells in a fibromyxoid stroma) that is very infrequent in other tumor types. A relevant question is whether there are generic elements altered in all those patterns of invasion [28][29]. In this study, we have found an increase of the membranous-cytoplasmic Ccnd1 expression in cells from specific invasion patterns (single cell/small cell clusters, buds, glandular) in which tumor cells are in contact with the adjacent stroma. More interestingly, increased membranous-cytoplasmic Ccnd1 expression was seen in peripheral cells in comparison with inner cells, whenever cancer cells infiltrate the stroma as large cell masses (collective or pushing patterns). Moreover, membranouscytoplasmic Ccnd1 expression was seen in peculiar forms of invasion, such as the MELF pattern. Considering all these data, we hypothesize that the presence of membranous-cytoplasmic Ccnd1 is a generic indicator of invasive phenotype.

We have also shown that forced attachment of Ccnd1 to the membrane (caused by Ccdn1-CAAX expression) induces tumor cell invasiveness and metastasis in tumor cells through a RalB-dependent mechanism. Considering all the data cited, we propose that the localization of Ccnd1 in the cytoplasm do not merely mean an appeasement of proliferation but could be a device to induce migration and invasion. Certainly, the role of Ccnd1 in the regulation of cell migration and invasion is also dependent on nuclear functions. $\mathrm{Li}$ et al, 2006 showed that the transcriptional repression of the cytoskeleton regulators ROCK II and TSP-1 exerted by Ccnd1 is required for efficient fibroblasts migration. Then, our proposal should not be categorically understood like that Ccnd1 shows a dichotomy as an oncogene, proliferation versus invasion, that can be unveiled depending on the subcellular localization, nucleus versus cytoplasm, respectively. By immunohistochemistry, Ccnd1 shows a diverse pattern of localizations in cancer tissues and cytoplasmic accumulation does not mean nuclear exclusion and vice versa ([30]; our results). Thus, we can envisage that when a detectable amount of Ccnd1 moves to the cytoplasm may be suggestive of an increment in the invasive potential of the cells.

The knowledge of the subcellular pattern of Ccnd1 may be interesting to determine the proliferative and invasive ability of specific tumors. It is well accepted that Ccnd1 nuclear accumulation is indicative that the tumor has a significant mitotic activity. For instance, cytoplasmic localization of Ccnd1 is observed in low-grade prostate carcinomas with reduced Ki-67 positivity whereas nuclear Ccnd1 is associated with high degree and elevated Ki-67 [30]. Conversely, cytoplasmic expression of Ccnd1 has not been taken into account as an indicator of tumor status other than the non-proliferative state. Nevertheless, single cell and MELF pattern in endometrial carcinoma, budding in colon cancer and pT3 tumors of prostatic carcinomas each of them exhibiting membranous-cytoplasmic Ccnd1 expression, have been associated with aggressiveness $[31][32][33][34][35][36][37]$. Then, we propose that cytoplasmic Ccnd1 could be indicative of tumor cells with a high invasive potential.

\section{MATERIALS AND METHODS}

\section{Tumor tissue samples}

Tumor samples, classified following the most recent WHO criteria, included 55 cases of endometrioid endometrial carcinoma, 50 cases of colorectal adenocarcinoma, 50 cases of breast carcinoma, and 50 cases of prostatic carcinoma. They were obtained from the Surgical Pathology files of Hospital Universitari Arnau de Vilanova, and IRBLLEIDA Biobank in Lleida, Spain. A specific informed consent was obtained from each patient in accordance with the protocols approved by the Local 
Ethical Committee. Tumors had been fixed in formalin and embedded in paraffin. Selected sections and paraffin blocks containing the invasive front of the tumors into the adjacent tissue were retrieved. Several specific types of tumor invasion, depending of tumor site, were evaluated (Table 1). In prostatic cancer, Gleason grade (3, 4 and 5) was taken as indirect indicator of invasion, and pT3 prostatic cancers were selected as the subgroup exhibiting the most prominent invasive features, by infiltrating periprostatic tissues.

\section{Cell culture}

MFE and Ishikawa (IK) cells are from endometrial adenocarcinoma. R3327-5' rat prostate-tumor cells were kindly provided by M. Hendrix. HEK293T cells were obtained from the American Type Culture Collection. Cells were maintained at $37^{\circ} \mathrm{C}$ in a $5 \% \mathrm{CO}_{2}$ incubator, and grown in Dulbecco's modified Eagle's medium (DMEM) supplemented with 10\% Fetal Bovine Serum (FBS), $100 \mu \mathrm{g} / \mathrm{ml}$ penicillin/streptomycin and $2 \mathrm{mM}$ glutamine. Transient transfection of vectors was performed with Lipofectamine 2000 (Invitrogen) according to manufacturer's instructions. For lentivirus production, HEK293T cells were transfected with lentiviral expression vectors, envelope plasmid pVSV.G, and packaging plasmid $\mathrm{pHR}$ ' $82 \Delta \mathrm{R}$ at a 2:1:1 ratio.

\section{Expression vectors}

Human CCND1 wild type and CCND1 ${ }_{\mathrm{K} 112 \mathrm{E}}$ inactive allele were used to obtain an N-terminal 3xHA fusion under the UBI promoter in a lentiviral vector derived from pDSL (Invitrogen) or under the CMV promoter in a pCDNA3 vector. In these constructs the 3 ' end of the $\mathrm{K}$-Ras ORF containing the CAAX motif (GGC TGT GTG AAA ATT AAA AAA TGC ATT ATA ATG TAA) was fused to the 3' end of the CCND1 ORF. For RalB activity assay we performed an $\mathrm{N}$-terminal 3xHA fusion to human RalB (image 3880116) and cloned in a pCDNA3 vector. Details of all constructs are available upon request. The RALB MISSION shRNA TRCN0000072957 and the CCND1 MISSION shRNA TRCN0000026883, both cloned in a pLKO.1-puro, were obtained from Sigma.

\section{Immunofluorescence}

Briefly, cells were quickly washed in PBS and fixed in $4 \%$ paraformaldehyde for $15 \mathrm{~min}$ at room temperature. Fixed MFE cells or fibroblasts were permeabilized with $0.2 \%$ triton- $100 \mathrm{X}$ for $3 \mathrm{~min}$ at room temperature, and blocked with 3\% BSA. Primary antibodies were combined with adequate Alexa488 and/or Alexa594-labeled secondary antibodies (Molecular Probes) in PBS with
0.3\% BSA. Nuclei were stained with Hoechst (Sigma). Images were acquired using $60 \mathrm{X}$ objective in an Olympus FV1000 confocal system. The antibodies used were antiHA (rat monoclonal 3F10, green), anti-Ccnd1 (rabbit monoclonal EP12 Dako, green) and anti-RalA (mouse monoclonal, red).

\section{Protein fractionation and immunoblot}

Protein fractionation was performed with the Subcellular Protein Fractionation kit for cultured cells (Thermo Scientific-Pierce; 78840). Soluble fraction corresponds to a mixture of cytosolic and nuclear soluble fractions described in the supplier's instructions.

For immunoblot, protein samples were resolved by SDS-PAGE, transferred to PVDF membranes (Millipore), and incubated with primary antibodies antiCcnd1 (monoclonal DCS-6, BD Pharmigen), anti-Cdk4 (polyclonal C-22, sc-260), anti-HA (rat monoclonal 3F10, Roche), anti-TfR (monoclonal H68.4, Invitrogen), antiRalB (rabbit polyclonal, Cell Signaling), anti-PCNA (monoclonal PC10, Abcam) and anti-tubulin (monoclonal B-5-1-2 Sigma). Appropriate peroxidase-linked secondary antibodies (GE Healthcare UK Ltd) were detected using the chemiluminescent HRP substrate Immobilon Western (Millipore). Chemiluminescence was recorded with a ChemiDoc-MP imaging system (BioRad).

\section{Cell invasion assay}

We performed cell invasion assays in 6.5-mm filters of 8.0 pore size (Transwell, Corning). Filters were coated with Matrigel (reduced-factors, BD Biosciences) in the upper side. Then, cells $\left(5 \times 10^{4}\right)$ were seeded in the bottom side of the filter for four hours to allow their attachment. Afterwards, filters were loaded with DMEM 10\% serum and incubated in 24-well plates containing serum-free medium for 24 hours. Under these conditions, some cells migrate from the bottom to the upper side of the filter invading the Matrigel. All cells were fixed and stained with Hoescht. Remaining cells at the bottom of the filter were removed and Matrigel-embedded cells were counted.

\section{Ral pull-down assay}

The Ral activation was analyzed by measuring the GTP-bound form of Ral. The assays were performed by using RalBP1 agarose (Upstate, cat\# 14-415) according to the manufacturer's instructions. Cell lysates were obtained from one $100 \mathrm{~mm}$ plate from transfected HEK293T cells. The lysis buffer used was $50 \mathrm{mM}$ Tris $\mathrm{pH} 7.5,200 \mathrm{mM} \mathrm{NaCl}, 2.5 \mathrm{mM} \mathrm{MgCl}, 2.5 \mathrm{mM}$ DTT, $1 \%$ Triton and protease and phosphatase inhibitors. $0.6 \mathrm{ml}$ of cell lysate was incubated with $10 \mu \mathrm{g}$ of RalBP1 beads 
during $30 \mathrm{~min}$ at $4^{\circ} \mathrm{C}$ and, after several washes, agarose beads were resuspended in 2x Laemmli buffer. Samples were separated by SDS-PAGE, transferred to PVDF membranes, and immunoblotted.

\section{Metastasis assay}

The procedure performed in this study followed the National Institutes of Health Guidelines for the Care and Use of Laboratory Animals, and was compliant with the guidelines of our Institution (UdL). Immunodeficient female SCID $\mathrm{hr} / \mathrm{hr}$ mice (8-week-old) were maintained in Specific Pathogen Free (SPF) conditions, and were inoculated with $5 \times 10^{5}$ MFE cells by retroorbital intravenous injection. Animals were sacrificed four weeks afterwards. For the metastasis assays injecting R3327-5'A cells, we have used fewer cells $\left(2.5 \times 10^{4}\right)$ and analyzed the lungs one week after injection, as those cells are very aggressive inducing metastasis.

\section{Immunohistochemistry}

Paraffin blocks of human tumor tissue samples were sectioned at a thickness of $3 \mu \mathrm{m}$, dried for 1 hour at $65^{\circ} \mathrm{C}$ before deparaffinization, rehydration, and epitope retrieval in the Pre- Treatment Module, PT LINK (Dako, Glostrup, Denmark) at $95^{\circ} \mathrm{C}$ for 20 minutes in $50 \AA \sim$ Tris/EDTA buffer, $\mathrm{pH}$ 9. Before staining the sections, endogenous peroxidase was blocked. Samples were subjected to IHC for Ccnd1 (1:25, EP12, DAKO), visualized with the EnVision FLEX Detection Kit (Dako, Glostrup, Denmark) using diaminobenzidine chromogen as a substrate. Slides were counterstained with hematoxylin. Negative controls were obtained without the addition of the primary antibody. The immunohistochemical analysis was conducted by pathologist and a researcher to ensure a preestablished histological criteria. IHC staining was assessed using ImageJ formal manual count method, $1,47 \mathrm{v}$ by Wayne Rasband (National Institutes of Health, USA), by analyzing tumor cells in a mean number of 5 invasive foci per case. Results indicate proportion of cells positively stained in the cytoplasm and membrane of selected fields (approximately 50 high resolution fields, X40). In the collective and pushing patterns, we assessed the difference in positively stained cytoplasm and membrane between peripheral and inner cells. Table 1 presents all invasion types evaluated in each of the different tumours studied.

Regarding mouse tissues, lung tissue samples were fixed with PFA. Blocks were sectioned at a thickness of $3 \mu \mathrm{m}$ and dried for $1 \mathrm{~h}$ at $65^{\circ} \mathrm{C}$, before being dewaxed in xylene and rehydrated through a graded ethanol series, then washed with PBS. Antigen retrieval was performed by heat treatment in a pressure cooker for $2 \mathrm{~min}$ in EDTA ( $\mathrm{pH}$ 8.9). Before staining the sections, endogenous peroxidase was blocked. The antibody used was anti-HA
12CA5 mouse monoclonal (ascitic fluid). After incubation, the reaction was visualized with the EnVision Detection Kit (Dako), using diaminobenzidine chromogen as a substrate. Sections were counterstained with hematoxylin.

\section{Statistical analysis}

For IHC data, mean and standard deviation were computed to assess the positivity observed in the cytoplasm for each tumoral type (endometrium, breast, prostate and colon) when comparing different invasion types or histological grades. Linear mixed models were used to evaluate the significance of the differences, using a random effect to take into account the samples corresponding to the same individual. All analyses were made using $\mathrm{R}$, setting the threshold for significance at 5\% $($ alfa $=0.05)$.

For cell data, comparisons among groups were made by one way ANOVA and Holm T-statistic post-test ( ${ }^{*} p<$ $0.05, * * p<0.01$, ns no significant).

For animal studies we have used the Ene 3.0: Program to calculate sample size. This software was developed by the Department of Applied Statistics of Autonomous University of Barcelona and is distributed by GlaxoSmithKline. No specific method of randomization was used but the group allocation was done randomly. Significance values were determined by Mann-Whitney U-Test Calculator (significance values 0.05 and two-tailed test).

\section{ACKNOWLEDGMENTS AND FUNDING}

We are grateful to M. Hendrix and E. Seftor for R3327-5'A cells. We thank Sònia Rius for technical assistance and members of the CYC and PATONC labs for helpful considerations. This work was funded by Spanish Ministry of Education and Science (BFU2010-20293/ BMC and BFU2013-42895-P), Catalan Government (SGR-559), and Fondo de Investigaciones Sanitarias (PI10/00604 and PI13/00263). X.M-G was supported by grants 2014SGR138, RD12/0036/0013, and Fundación Asociación Española contra el Cancer. Tumour samples were obtained with the support of Xarxa Catalana de Bancs de Tumors, and Plataforma de Biobancos ISCIII (PT13/0010/0014). NP. Fusté was supported by a contract from "Fundació Alicia Cuello de Merigó". I. Felip and T. Cemeli were supported by a predoctoral fellowship from FPU-MINECO.

\section{CONFLICTS OF INTEREST}

The authors declare that they have no conflict of Interest. 


\section{Authors' contributions}

E.C., M.S., J.P., S.G. and X. M-G performed and analyzed pathology data. NP.F., I.F., R.F-H.,T.C., E.G., M.T., and X.D. performed constructions, in vitro and mice experiments. F.F. and N.P. provided cell-culture support, contributed materials and helped with procedures. X. M-G, X.D., and E.G. designed the project and analyzed the data. X. M-G, X.D., and E.G. wrote the manuscript. All authors contributed discussions and comments on the manuscript.

\section{REFERENCES}

1. Beroukhim R, Mermel CH, Porter D, Wei G, Raychaudhuri S, Donovan J, Barretina J, Boehm JS, Dobson J, Urashima M, Mc Henry KT, Pinchback RM, Ligon AH, et al. The landscape of somatic copy-number alteration across human cancers. Nature. 2010; 463:899-905.

2. Motokura T, Bloom T, Kim HG, Jüppner H, Ruderman J V, Kronenberg HM, Arnold A. A novel cyclin encoded by a bcl1-linked candidate oncogene. Nature. 1991; 350:512515.

3. Xiong Y, Connolly T, Futcher B, Beach D. Human D-type cyclin. Cell. 1991; 65:691-699.

4. Wang TC, Cardiff RD, Zukerberg L, Lees E, Arnold A, Schmidt E V. Mammary hyperplasia and carcinoma in MMTV-cyclin D1 transgenic mice. Nature. 1994; 369:669671.

5. Matsushime H, Ewen ME, Strom DK, Kato JY, Hanks SK, Roussel MF, Sherr CJ. Identification and properties of an atypical catalytic subunit (p34PSK-J3/cdk4) for mammalian D type G1 cyclins. Cell. 1992; 71:323-334.

6. Kato J, Matsushime H, Hiebert SW, Ewen ME, Sherr CJ. Direct binding of cyclin D to the retinoblastoma gene product $(\mathrm{pRb})$ and $\mathrm{pRb}$ phosphorylation by the cyclin D-dependent kinase CDK4. Genes and Development. 1993; 7:331-342.

7. Baldin V, Lukas J, Marcote MJ, Pagano M, Draetta G. Cyclin D1 is a nuclear protein required for cell cycle progression in G1. Genes \& Development. 1993; 7:812821.

8. Sherr CJ, Roberts JM. Living with or without cyclins and cyclin-dependent kinases. Genes \& Development. 2004; 18:2699-711.

9. Ewen ME, Lamb J. The activities of cyclin D1 that drive tumorigenesis. Trends in Molecular Medicine. 2004; 10:158-162.

10. Kim JK, Diehl JA. Nuclear cyclin D1: an oncogenic driver in human cancer. Journal of Cellular Physiology. 2009; 220:292-6.

11. Drobnjak M, Osman I, Scher HI, Fazzari M, CordonCardo C. Overexpression of cyclin D1 is associated with metastatic prostate cancer to bone. Clinical Cancer
Research. 2000; 6:1891-5.

12. Huang $\mathrm{H}, \mathrm{Hu} \mathrm{Y}, \mathrm{Li} \mathrm{N}, \mathrm{Zhu} \mathrm{Y}$. Inhibition of tumor growth and metastasis by non-small cell lung cancer cells transfected with cyclin D1-targeted siRNA. Oligonucleotides. 2009; 19:151-62.

13. Neumeister P, Pixley FJ, Xiong Y, Xie H, Wu K, Ashton A, Cammer M, Chan A, Symons M, Stanley ER, Pestell RG. Cyclin D1 governs adhesion and motility of macrophages. Molecular Biology of the Cell. 2003; 14:2005-2015.

14. Li Z, Wang C, Jiao X, Lu Y, Fu M, Quong A a, Dye C, Yang J, Dai M, Ju X, Zhang X, Li A, Burbelo P, et al. Cyclin D1 regulates cellular migration through the inhibition of thrombospondin 1 and ROCK signaling. Molecular and Cellular Biology. 2006; 26:4240-56.

15. Li Z, Chen K, Jiao X, Wang C, Willmarth NE, Casimiro MC, Li W, Ju X, Kim SH, Lisanti MP, Katzenellenbogen JA, Pestell RG. Cyclin D1 integrates estrogen-mediated DNA damage repair signaling. Cancer Research. 2014; 74:3959-70.

16. Zhong Z, Yeow W-S, Zou C, Wassell R, Wang C, Pestell RG, Quong JN, Quong A a. Cyclin D1/cyclin-dependent kinase 4 interacts with filamin $\mathrm{A}$ and affects the migration and invasion potential of breast cancer cells. Cancer Research. 2010; 70:2105-14.

17. Alhaja E, Adan J, Pagan R, Mitjans F, Cascalló M, Rodríguez M, Noé V, Ciudad CJ, Mazo A, Vilaró S, Piulats J. Anti-migratory and anti-angiogenic effect of p16: a novel localization at membrane ruffles and lamellipodia in endothelial cells. Angiogenesis. 2004; 7:323-33.

18. Fernández RMH, Ruiz-Miró M, Dolcet X, Aldea M, Garí E. Cyclin D1 interacts and collaborates with Ral GTPases enhancing cell detachment and motility. Oncogene. 2011; 30:1936-46.

19. Meng H, Tian L, Zhou J, Li Z, Jiao X, Li WW, Plomann M, Xu Z, Lisanti MP, Wang C, Pestell RG. PACSIN 2 represses cellular migration through direct association with cyclin D1 but not its alternate splice form cyclin D1b. Cell Cycle. 2011; 10:73-81.

20. Fleischmann A, Rocha C, Saxer-Sekulic N, Zlobec I, Sauter G, Thalmann GN. High-level cytoplasmic cyclin D1 expression in lymph node metastases from prostate cancer independently predicts early biochemical failure and death in surgically treated patients. Histopathology. 2011; 58:781-789.

21. Wolthuis RM, de Ruiter ND, Cool RH, Bos JL. Stimulation of gene induction and cell growth by the Ras effector Rlf. The EMBO Journal. 1997; 16:6748-61.

22. Hancock JF. Ras proteins: different signals from different locations. Nature Reviews Molecular Cell Biology. 2003; $4: 373-384$.

23. Biliran H, Wang Y, Banerjee S, Xu H, Heng H, Thakur A, Bollig A, Sarkar FH, Liao JD. Overexpression of cyclin D1 promotes tumor cell growth and confers resistance to cisplatin-mediated apoptosis in an elastase-myc transgene- 
expressing pancreatic tumor cell line. Clinical Cancer Research. 2005; 11:6075-6086.

24. Ikeda Y, Oda K, Hiraike-Wada O, Koso T, Miyasaka A, Kashiyama T, Tanikawa M, Sone K, Nagasaka K, Maeda D, Kawana K, Nakagawa S, Fukayama M, et al. Cyclin D1 harboring the T286I mutation promotes oncogenic activation in endometrial cancer. Oncology Reports. 2013; 30:584-588.

25. Solomon DA, Wang Y, Fox SR, Lambeck TC, Giesting S, Lan Z, Senderowicz AM, Knudsen ES. Cyclin D1 splice variants: Differential effects on localization, RB phosphorylation, and cellular transformation. Journal of Biological Chemistry. 2003; 278:30339-30347.

26. Lu F, Gladden AB, Diehl JA. An Alternatively Spliced Cyclin D1 Isoform, Cyclin D1b, Is a Nuclear Oncogene. Cancer Research. 2003; 63:7056-7061.

27. Zheng L, Qi T, Yang D, Qi M, Li D, Xiang X, Huang K, Tong Q. microRNA-9 suppresses the proliferation, invasion and metastasis of gastric cancer cells through targeting cyclin D1 and Ets1. PloS One. 2013; 8:e55719.

28] Montserrat N, Mozos A, Llobet D, Dolcet X, Pons C, de Herreros AG, Matias-Guiu X, Prat J. Epithelial to mesenchymal transition in early stage endometrioid endometrial carcinoma. Human Pathology. 2012; 43:632643.

29. Monge M, Doll A, Colas E, Gil-Moreno A, Castellvi J, Garcia A, Colome N, Perez-Benavente A, Pedrola N, Lopez-Lopez R, Dolcet X, Ramon Y Cajal S, Xercavins $\mathrm{J}$, et al. Subtractive proteomic approach to the endometrial carcinoma invasion front. Journal of Proteome Research. 2009; 8:4676-4684.

30. Comstock CES, Revelo MP, Buncher CR, Knudsen KE. Impact of differential cyclin D1 expression and localisation in prostate cancer. British Journal of Cancer. 2007; 96:970 979.
31. Stewart CJR, Brennan BA, Leung YC, Little L. MELF pattern invasion in endometrial carcinoma: association with low grade, myoinvasive endometrioid tumours, focal mucinous differentiation and vascular invasion. Pathology. 2009; 41:454-459.

32. Euscher E, Fox P, Bassett R, Al-Ghawi H, Ali-Fehmi R, Barbuto D, Djordjevic B, Frauenhoffer E, Kim I, et al. NIH Public Access. Am J Surg Pathol. 2013; 37:1728-1736.

33. Hertel JD, Huettner PC, Pfeifer JD. Lymphovascular space invasion in microcystic elongated and fragmented (MELF)pattern well-differentiated endometrioid adenocarcinoma is associated with a higher rate of lymph node metastasis. International Journal of Gynecological Pathology. 2014; 33:127-34.

34. Wang LM, Kevans D, Mulcahy H, O'Sullivan J, Fennelly D, Hyland J, O'Donoghue D, Sheahan K. Tumor budding is a strong and reproducible prognostic marker in T3N0 colorectal cancer. The American Journal of Surgical Pathology. 2009; 33:134-141.

35. Betge J, Kornprat P, Pollheimer MJ, Lindtner RA, Schlemmer A, Rehak P, Vieth M, Langner C. Tumor Budding is an Independent Predictor of Outcome in AJCC/ UICC Stage II Colorectal Cancer. Annals of Surgical Oncology. 2012; 19:3706-3712.

36. Lai Y-H, Wu L-C, Li P-S, Wu W-H, Yang S-B, Xia P, He $\mathrm{X}-\mathrm{X}$, Xiao L-B. Tumour budding is a reproducible index for risk stratification of patients with Stage II colon cancer. Colorectal Disease. 2014; 16:259-64.

37. Salhia B, Trippel M, Pfaltz K, Cihoric N, Grogg A, Lädrach C, Zlobec I, Tapia C. High tumor budding stratifies breast cancer with metastatic properties. Breast Cancer Research and Treatment. 2015; 150:363-71. 